\title{
Thermographic Signal Reconstruction with periodic temperature variation applied to moisture classification
}

\author{
by P. Bison, G. Cadelano and E. Grinzato
}

\author{
CNR-ITC, C.so Stati Uniti 4, 35127 Padova, Italy, paolo.bison@itc.cnr.it
}

\begin{abstract}
A method to analyse thermographic data with periodic temperature variation is proposed. A periodic heat flux is generated on the surface of a specimen with a non-zero average value. Functions describing the periodic temperature response plus the transient effect are considered. A possible linear trend is also taken into account aiming at considering slow environmental variations. Based on the superposition of the effects, thanks to the linearity of the heat conduction equation, the analysis of the thermographic signal is proposed that applies to the classification of the evaporative effects in moistened porous building materials.
\end{abstract}

\section{Introduction}

Several techniques have been proposed to enhance the temperature signal coming from pulsed thermographic sequences. The TSR (Thermal Signal Reconstruction) [1] is known for its peculiar transformation in log-log space where the temperature response of a semi-infinite body is a straight line. The successive polynomial fitting, provided by the technique, is mainly the description of the departures of the object under test from the semi-infinite model. It is named TSR for the capability to reconstruct the temperature signal from few polynomial coefficients with great compression capabilities and noise reduction. Very popular is the PPT (Pulsed Phase Thermography) [2] also. It is well known that a suitable superposition of sines and cosines could approximate almost any function. PPT is based on the Discrete Fourier Transform that permits to represent the original signal by means of amplitude and phase of its components in the frequency domain. Particular interest is drawn by the phase maps that are less sensitive to any multiplicative factor affecting surface temperature as optical properties varying of the object under test. PCA (Principal Component Analysis) [3] is similar to PPT in so far it is able to produce a dual space of functions whose superposition gives back the original temperature signal. In this case the basic function are not known a priori as for PPT but are built on the base of the criterion of the maximization of the variance in the temperature signal, with the constraint to represent an orthogonal system of functions as for PPT. A summary of these algorithms is given in [4]. More recently the TWSR (Thermal Waves Signal Reconstruction) [5] has been proposed to enhance the defects contrast in lock-in thermography experiments.

The application that gives rise to this work is the classification of moisture content in porous material, mainly used in buildings [6], by IR thermography.

Monitoring the moisture content of a wall is a very important issue, even if limited to the shallow layer, close to the surface that correspond normally to the plaster. It is a matter of fact that the moisture excess highly contributes to the decay of building. Actually, most of the buildings are affected by such a problem. It is well known that the evaporation process, with the migration of salts within the material, represents the main reason for the deterioration of the surfaces [7]. Tensions and stresses due to salts crystallisation on the surface and the mechanical stress and micro cracks due to icing-thawing cycles are very dangerous. Chemical and physical phenomena are also activated by moisture, as the growing of bacteria, algae, fungi and lichens. Many methods have been proposed for this purpose, but up today the ultimate solution is far to be found. The measurement of the moisture content is difficult because it is an indirect measurement using as informative parameters quantities such as: the mass, the electrical conductivity, the electromagnetic wave absorption in specific bands, the propagation speed of elastic waves, the dielectric constant of material or the magnetic resonance of hydrogen atoms. Among these methods, temperature is also a valid indicator of the presence of moisture and humidity. However, the complexity of the structure of a building, and the dependence of its thermal history from the climatic conditions and from the use for which it is intended for, make complex the interpretation of data and not always reliable. This is particularly true when thermographic surveys are performed where the boundary conditions, as the sun or the wind, make very variable the surface temperature, both in time and space domain. Nevertheless, IR thermography is a valid tool to locate moist area, because the water content of a porous material is strongly related to the surface temperature. Unfortunately, the surface temperature itself is not sufficient to solve the inverse heat and mass transfer problem. This issue is worthy of further consideration, because the vapour condensation-evaporation affects significantly the heat flux and vice versa. Therefore, the reliability of findings is questionable in many cases. On the other side, such an optical testing is very productive and definitively non destructive. These features make it as the most suitable for inspecting very large surfaces. The main characteristic of this approach is to take advantage of the optical nature of the thermographic device, that is an imaging device. 
The underlying idea for the improved thermographic detection of moisture is that of enhancing the water evaporation from the surface of the material, by means of an increased air speed [8]. In such a way, only the moist surface is cooled and the temperature locally varies on it. The temperature variation should be higher on the surface of the material with higher moisture content, while it should not influence the dry surface, provided that the air that is flowing has the same temperature of the dry material.. It is also supposed that materials with different moisture content exhibits a different time response in term of temperature decreasing, with amplitudes and time constant proportional to the moisture content. In order to better differentiate the experimental forced temperature response present in any moistened porous material from that naturally due to the environment, it is planned to submit the material to a continuously modulated ventilation with varying air velocity, from zero to a fixed value (of the order of some $\mathrm{m} / \mathrm{s}$ ). The velocity is modulated in an approximately sinusoidal way. As a consequence of the ventilation, the surface temperature of the moistened material varies periodically at the period of the forcing ventilation and, at the same time, it decreases in the mean as a consequence of the fact that the stimulus is generating a heat flux (due to the latent heat extraction) with a negative average value.

Here a linear fit of temperature, periodically varying with known period, by sine and cosine components, permits to extract phase and amplitude maps. Moreover a polynomial fitting is added to the periodic one. That permits to follow the temperature signal even in the initial part of the experiment, when the steady periodic regime is not yet reached and the mean value of the sine and cosine is therefore changing with time. The technique of linear fitting in lock-in thermography is considered the best compromise according to Krapez [9] in comparison with algorithms like the standard lock-in, the 4-buckets and the variance method. The present work is based on the idea exposed in [5] and [9], with an enhancement that relies on the a-priori assumption on the kind of transient phenomena affecting the periodic signal.

\section{Rationale}

It is supposed that the evaporation enhancement on the surface of the moistened sample, due to the variation of the air speed, increases the latent heat extraction due to the phase change of water. That can be modeled by a source of negative heat flux that varies according to the air velocity that flows on the surface. As the air velocity is modulated periodically, the sample under test is submitted to a periodic negative heat flux:

$$
\Phi=-\Phi_{0}(1+\sin (\omega t+\varphi))
$$

where $\Phi_{0}$ is a constant depending on the air velocity and on the amount of moisture in the sample, $t$ is time, $\omega=2 \pi f$ is the angular frequency ( $f$ being the frequency) and $\varphi$ the initial phase of the periodic function. If the air velocity flowing on the surface of the object is the same in every point, the heat flux sink depends only on the amount of moisture present in every part of the surface. The greater the moisture the greater the heat sink and consequently the lowering of temperature.

Equation 1 appears to be composed of a constant part and a periodic one. By the superposition principle the temperature response will be the sum of the step function response plus the periodic behaviour [10]. Here it is neglected the transient effect given by the initial phase of the periodic function that disappears after few periods. The step function response is proportional to the square root of time if a sufficiently thick sample is considered and the experiment duration is short enough that the sample could be approximated by a semi-infinite body. The periodic part is well described by a superposition of sine and cosine with suitable coefficients. If more harmonic component are present in the signal, successive harmonic components could be considered. Moreover it is taken into account a possible slow trend in the laboratory conditions such that it could be described by a simple linear function. With such assumptions the temperature of each pixel in the thermographic sequence is well approximated by a function such that:

$$
T_{i, j}(t)=A 0_{i, j}+A 1_{i, j} t+A 2_{i, j} \sqrt{t}+b 1_{i, j} \cos (\omega t)+c 1_{i, j} \sin (\omega t)
$$

where $T$ is temperature, $i, j$ are indexes of the pixel in the image matrix. The amplitude of the periodic part of the temperature function and its phase are given respectively by:

$$
\rho=\sqrt{b 1_{i, j}^{2}+c 1_{i, j}^{2}} \quad \psi=\arctan \left(\frac{c 1_{i, j}}{b 1_{i, j}}\right)
$$

and equation (2) can be rewritten as follows:

$$
T_{i, j}(t)=A 0_{i, j}+A 1_{i, j} t+A 2_{i, j} \sqrt{t}+\rho_{i, j} \cos \left(\omega t+\psi_{i, j}\right)
$$




\subsection{Numerical simulation}

In order to check the capabilities of equation 4 to model the process of modulated evaporation, the surface temperature profile is computed by the numerical solution of the 1D Partial Differential Equation:

$$
\frac{\partial^{2} T}{\partial x^{2}}-\frac{1}{\alpha} \frac{\partial T}{\partial t}=0
$$

with $x$ the spatial coordinate and $\alpha$ thermal diffusivity. The solution is given in case of a thick slab with boundary condition of prescribed heat flux on one surface (the back of the sample is considered adiabatic) and with varying heat conduction exchange coefficient, results of the varying velocity of air on the surface:

$$
\begin{aligned}
& -\left.\lambda \frac{\partial T}{\partial x}\right|_{x=0}=-\Phi_{0}(1+\sin (\omega t))-\left[h_{0}+h_{1}(1+\sin (\omega t))\right] \\
& \left.\lambda \frac{\partial T}{\partial x}\right|_{x=L}=0
\end{aligned}
$$

here $\lambda$ is the thermal conductivity of the material, $h_{0}$ is the heat exchange coefficient due to the natural convection and $h_{1}$ is the heat exchange coefficient of the forced convection that is modulated periodically, $L$ is the thickness of the slab. The initial conditions of the sample are of null temperature and the temperature of the environment is null as well. By means of the MATLAB ${ }^{\circledR}$ PDE solver [11], the equation 5 is computed with boundary as in equation 6 . The parameters used in the computation are reported in Table 1 . In particular the value of $\Phi_{0}$ is based on the value of the Latent Heat of water at $23{ }^{\circ} \mathrm{C}\left(\mathrm{LH}\left(\mathrm{T}=20^{\circ} \mathrm{C}\right) \sim 2.4 \cdot 10^{6} \quad \mathrm{~J} \mathrm{~kg}^{-1}\right)$ and on the Evaporation Rate experimentally obtained for a saturated Pietra Serena stone (ER $\left.\sim 3.5 \cdot 10^{-5} \mathrm{~kg} \mathrm{~m}^{-2} \mathrm{~s}^{-1}\right)$ in an environment with $45 \%$ of Relative Humidity and an air speed of $1.5 \mathrm{~m} \mathrm{~s}^{-1}$. The result of the simulation is presented in Fig. 1.

Table 1. Parameters of the simulation

\begin{tabular}{|c|c|c|c|c|c|c|c|c|c|}
\hline \multicolumn{4}{|c|}{ Material: Pietra Serena } & \multicolumn{3}{c|}{$\begin{array}{c}\text { Boundary conditions } \\
\text { parameters }\end{array}$} & \multicolumn{3}{c|}{ frequency } \\
\hline $\begin{array}{c}\lambda \\
\mathrm{Wm}^{-1} \mathrm{~K}^{-1}\end{array}$ & $\begin{array}{c}\rho \\
\mathrm{kg} \mathrm{m}^{-3}\end{array}$ & $\begin{array}{c}\mathrm{C}_{\mathrm{p}} \\
\mathrm{J} \mathrm{kg}^{-1} \mathrm{~K}^{-1}\end{array}$ & $\begin{array}{c}\alpha \\
\mathrm{m}^{2} \mathrm{~s}^{-1}\end{array}$ & $\begin{array}{c}\mathrm{L} \\
\mathrm{m}\end{array}$ & $\begin{array}{c}\Phi_{0} \\
\mathrm{Wm}^{-2}\end{array}$ & $\begin{array}{c}\mathrm{h}_{0} \\
\mathrm{Wm}^{-2} \mathrm{~K}^{-1}\end{array}$ & $\begin{array}{c}\mathrm{h}_{1} \\
\mathrm{Wm}^{-2} \mathrm{~K}^{-1}\end{array}$ & $\begin{array}{c}\mathrm{f} \\
\mathrm{s}^{-1}\end{array}$ & $\begin{array}{c}{ }^{1} \\
\mathrm{~s}^{-1}\end{array}$ \\
\hline 2.45 & 2540 & 811 & $1.2 \cdot 10^{-6}$ & 0.05 & 86 & 5 & 50 & 0.125 & 0.7854 \\
\hline
\end{tabular}

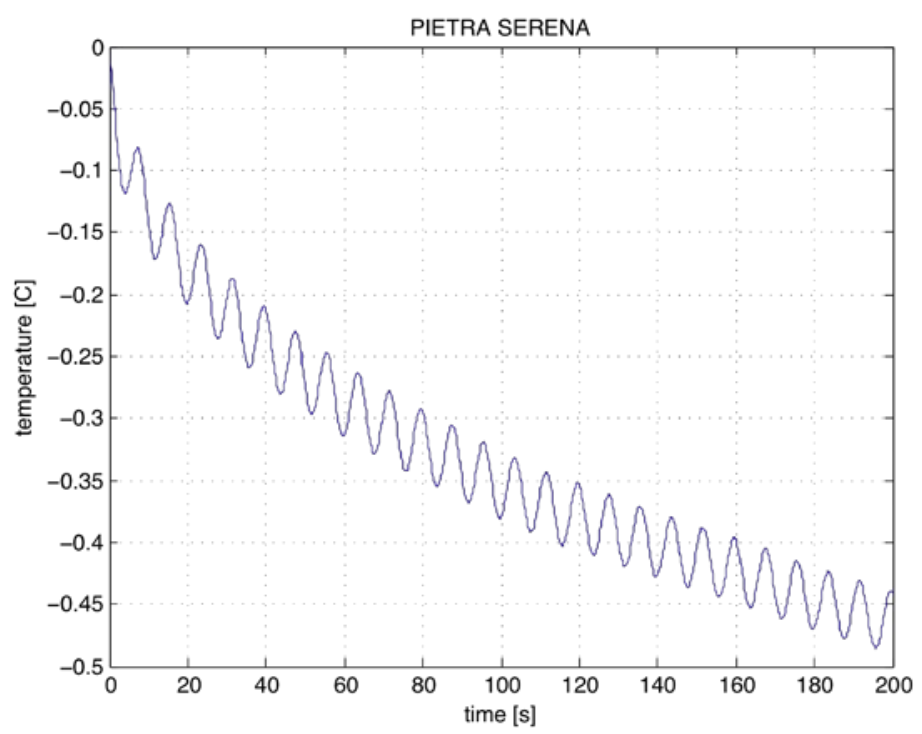

Fig.1. Result of the simulation: surface temperature profile of a moistened block of material.

Parameters are reported in Table 1. 


\subsection{Data reduction}

Even though equation 2 can not be able to completely follow the complicated behaviour depicted by the numerical simulation, it has the great advantage of being linear in the parameters and it could be easily used to fit the data by the linear least-square technique. The equation 2 could be conveniently written in matrix form in the following way:

$$
\mathbf{M}=\left(\begin{array}{ccccc}
1 & t_{1} & \sqrt{t_{1}} & \cos \left(\omega t_{1}\right) & \sin \left(\omega t_{1}\right) \\
1 & t_{2} & \sqrt{t_{2}} & \cos \left(\omega t_{2}\right) & \sin \left(\omega t_{2}\right) \\
1 & t_{N} & \sqrt{t_{N}} & \cos \left(\omega t_{N}\right) & \sin \left(\omega t_{N}\right)
\end{array}\right)
$$

$t_{1} \ldots t_{N}$ being the times of data acquisition. The parameters to be estimated correspond to a column vector $\mathbf{p}=\left(A_{0}, A_{1}, A_{2}, b_{1}, c_{1}\right)^{\prime}$, the prime meaning the transpose operation. Finally the experimental data can be arranged in a column vector as well $T=\left(T\left(t_{1}\right), T\left(t_{2}\right), \ldots, T\left(t_{N}\right)\right)^{\prime}$, with $T\left(t_{i}\right)$ the temperature collected at time $t_{i}$.

With this definitions the problem of estimating the parameters in the least-square sense is that of solving the system of linear equations given by:

$$
\mathbf{M} \cdot \mathbf{p}=\mathbf{T}
$$

where the equality must be intended in the approximation of the least-squares criterion. In such a case the solution is given by the normal equation that in matrix form is given by:

$$
\mathbf{p}=\left(\mathbf{M}^{\prime} \cdot \mathbf{M}\right)^{-1} \cdot \mathbf{M}^{\prime} \cdot \mathbf{T}
$$

With this formulation the problem of determining the fitting parameters is easily solved by means of standard routine present in any package of linear problem analysis. To test the algorithm, the data obtained by the simulation process and shown in Fig. 1 are slightly corrupted by adding noise with normal distribution and standard deviation equal to $0.01{ }^{\circ} \mathrm{C}$ typical of an IR camera. The data are fitted by the equation 9 and the results are shown in Fig. 2.

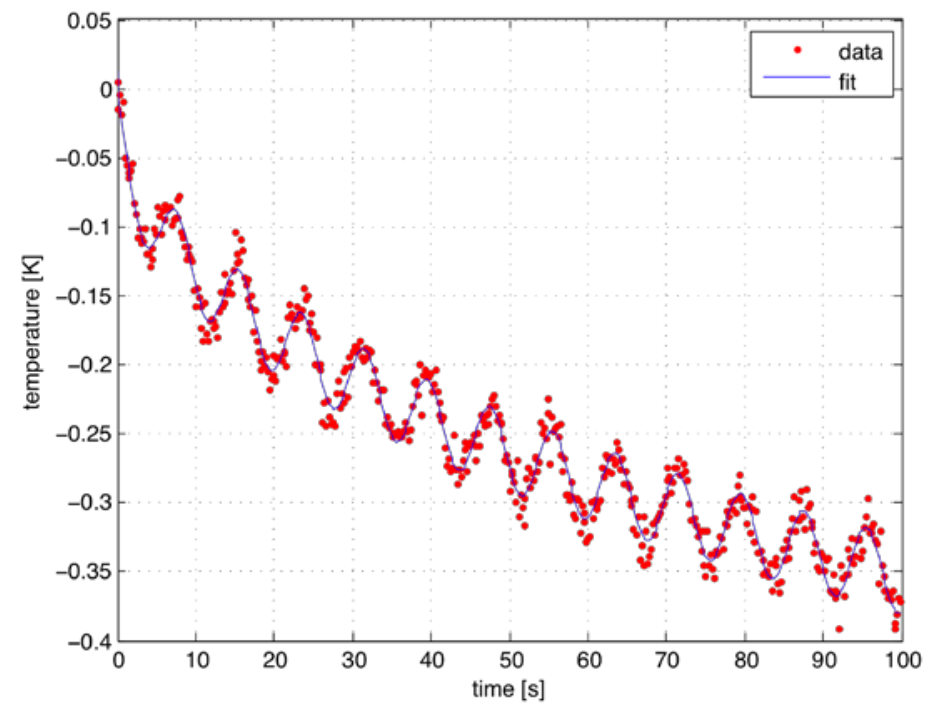

Fig.2. Fitting of data by equation 2. Data are obtained by simulation and corrupted with $0.01{ }^{\circ} \mathrm{C}$ of random noise with normal distribution.

\section{Experiment}

Some samples of bricks, plasters and stones are considered and conditioned at various degree of water content. The samples are submitted to ventilation with air velocity varying from zero to a certain maximum value, with period $8 \mathrm{~s}$. The air ventilation increases the evaporation from the surface of the specimens extracting latent heat. The effect on temperature is that of cooling down the surface of the specimens and due to the periodic velocity variation it leads to a periodic temperature variation with a net decreasing due to the non zero average value of the heat sink. 
In Fig. 3 it is represented the IR image with several specimen of different materials and moistened with different degree of water content. Three areas are indicated with numbers 1, 2 and 3. The description of the samples and the degree of water content is reported in Table 2.

Table 2. Areas indicated in Fig. 3: correspondence with kind of material and water content

\begin{tabular}{|l|l|l|}
\hline area number & material & water content \\
\hline$\# 1$ & Pietra Serena stone & high \\
\hline$\# 2$ & Plaster & medium-high \\
\hline$\# 3$ & Pietra Serena stone & dry \\
\hline
\end{tabular}

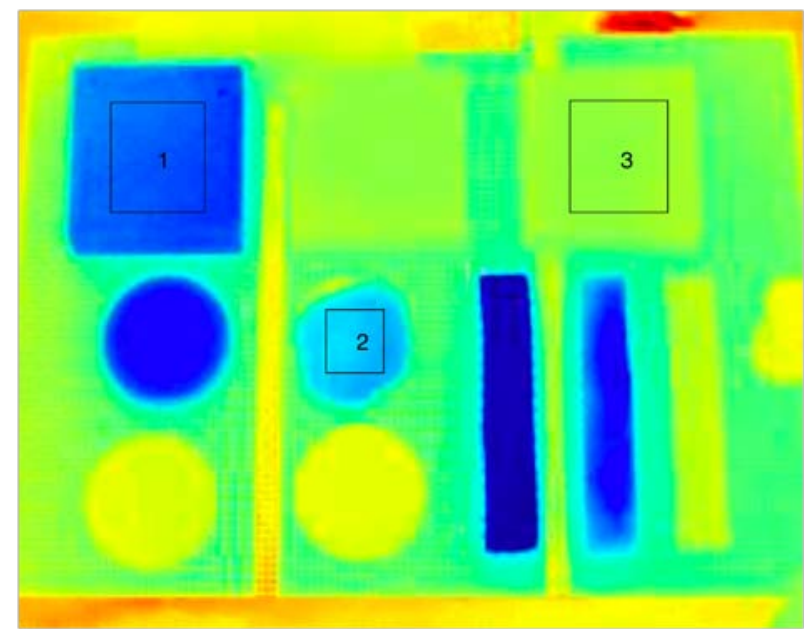

Fig. 3. IR image with different samples moistened with different water contents. Three areas are indicated: correspondence is reported in Table 2.

In correspondence of the areas indicated in Fig. 3 the temperature plots are reported in Fig. 4. Notice the low variation of Pietra Serena stone and the high decreasing of temperature together with a clear oscillation of the plaster specimen. Finally, in correspondence of the dry Pietra Serena specimen the temperature variation is negligible. The periodic ventilation is stopped after $120 \mathrm{~s}$ from the starting of the experiment. The time is indicated by a vertical black line in Fig. 4. Notice how the temperatures of the samples asymptotically return to the values of the beginning of the experiment. It is also to notice how different are the temperature values at the beginning of the experiment. Each sample, depending on the water content, on its porosity, on its surface finishing and thermal properties reaches different equilibrium temperatures with the environment. In Fig. 5 the maps representing the various coefficients described in eq. 2 are represented.

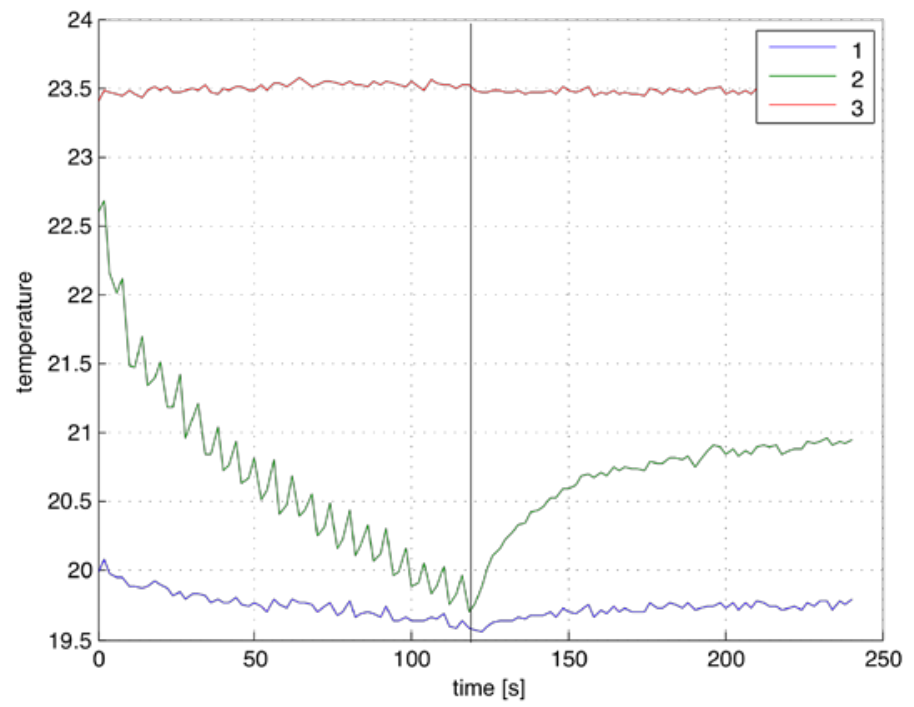

Fig. 4. Temperature plot of the three areas indicated in Fig. 3. 


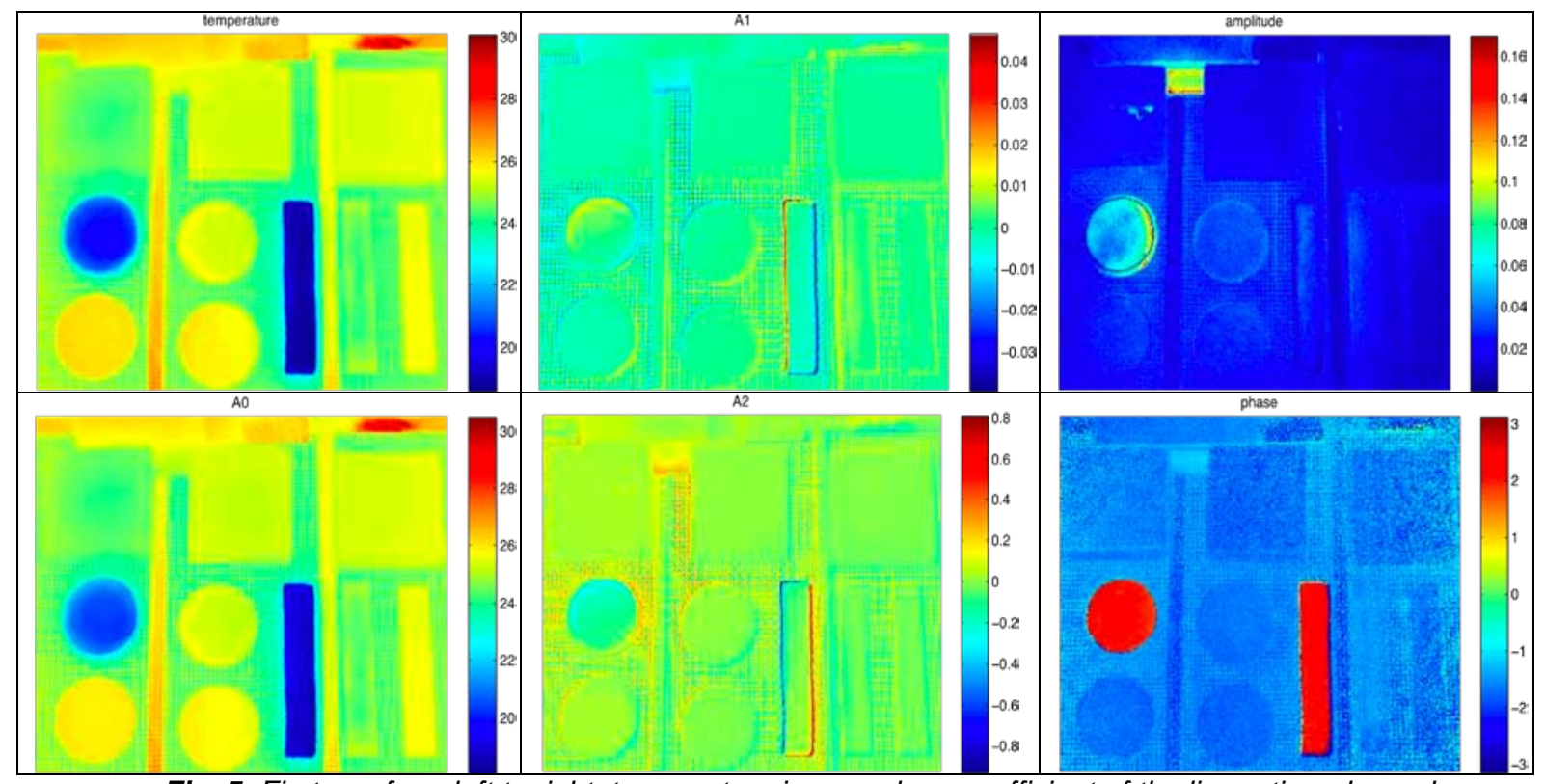

Fig. 5. First raw from left to right: temperature image; slope coefficient of the linear time dependence; amplitude of the harmonic component at 8s period. Second raw from left to right: intercept of the linear time dependence; coefficient of the square root of time dependence; phase of the harmonic component at $8 \mathrm{~s}$ period.

Further experiments at $16 \mathrm{~s}$ period of periodic ventilation was carried out. Table 3 shows the level of moisture content for each material. Such a value is correlated to the porosity of the bulk material.

Table 3. moisture content of each tested material and degree of saturation. 'sat':saturated; 'int':internediate.

\begin{tabular}{|c|c|c|c|c|c|c|c|c|c|c|}
\hline $\begin{array}{c}\text { Sample } \\
\text { code }\end{array}$ & $\mathrm{P} 1$ & $\mathrm{P} 2$ & $\mathrm{P} 3$ & $\mathrm{M} 1$ & $\mathrm{M} 2$ & $\mathrm{M} 3$ & $\mathrm{I}$ & $\mathrm{I}$ & $\mathrm{I}$ & 14 \\
\hline condition & sat & int & dry & sat & int & dry & sat & int & dry & int \\
\hline $\begin{array}{c}\text { Moisture } \\
\text { content }\end{array}$ & $2 \%$ & $0,5 \%$ & $0,001 \%$ & $25 \%$ & $0,2 \%$ & $0,001 \%$ & $63 \%$ & $23 \%$ & $0,05 \%$ & $5 \%$ \\
\hline
\end{tabular}

Figure 6a shows the tested materials with indication of the air temperature, relative humidity and air velocity probe while Figure $6 \mathrm{~b}$ shows the corresponding thermogram. Finally, Figure 7 shows the plot of surface temperature in correspondence of the various samples, during the experiment. 



Fig.6a. Arrangements of the tested materials, with indication of air temperature, relative humidity ('^':saturated; '=': intermediate; ' $\vee$ ': dry) and air velocity probes 'va'



Fig.7. plot vs. time of the mean surface temperature of each sample; the vertical dotted line indicates the end of the forced ventilation

\section{Conclusions}

The paper presents an effective data reconstruction technique, describing an oscillating heat flux, coupled with a diffusion process and a trend in the boundary conditions, all of these affecting the surface temperature. A compact mathematical form is presented, suitable to process sequences of thermographic images.

Such a technique has been applied to study the moisture detection by IR thermography on buildings. This approach has been used to verify the simplified thermal model underlying the hypothesis that "the greater the moisture the greater the heat sink effect due to the evaporation" and consequently the lowering of temperature. Unfortunately, the evaporation rate has a complex and not linear behaviour with the moisture content. An interesting result of this technique is the possibility to measure the actual distance from a simplified model as that described and experimental data. The following of this project is to improve the model, taking into account a more complex mathematical description. What seems to be demonstrated is the validity of the mathematic synthesis of the data for diffusive process including phase change. A good agreement with 
experiments has been found for both high and low moisture content, in the intermediate range a more deep understanding and experimental work has to be carried out.

\section{REFERENCES}

[1] Shepard S.M., "Advances in Pulse Thermography," SPIE Proceedings Vol. 4360, Thermosense XXIII, pp. 511-515, 2001.

[2] Maldague X. and Marinetti S., "Pulse Phase Infrared Thermography," J. Appl. Phys., 79 (5), pp. 26942698, 1996.

[3] Rajic N.,"Principal component thermography for flaw contrast enhancement and flaw depth characterisation in composite structures," Composite Structures, 58, pp. 521-528, 2002.

[4] Ibarra-Castanedo C., Gonzalez D., Klein M., Pilla M., Vallerand S. and Maldague X.," Infrared image processing and data analysis," Infrared Physics \& Technology, 46, pp. 75-83, 2004.

[5] Quek S., Almond D., Nelson L. and Barden T., "A Novel and Robust Thermal Wave Signal Reconstruction Technique for Defect Detection in Lock-In Thermography," Meas. Sci. Technol., 16 (5), pp. 1223-1233, 2005.

[6] Grinzato E., Bison P.G., Marinetti S. and Vavilov V., "Thermal/Infrared nondestructive evaluation of moisture content in building: theory and experiment", in Proceedings of the international symposium "Dealing With Defects In Building", Varenna (Italy), Eds. M Moroni and P. Sartori, September 1994, Part 1, 1994, pp. 345-356.

[7] Grinzato E., Cadelano G., Bison P., "Moisture map by IR thermography" Journal of Modern Optics, Taylor \& Francis (in press, 2010)

[8] Massari G. and M. Massari M., "Damp buildings, old and new", Rome: ICCROM, 1993.

[9] Krapez J.C., "Compared performances of four algorithms used for modulation thermography," Proceedings of Eurotherm Seminar n. 60, QIRT 98, pp. 148-153, 1998.

[10] Carslaw H.S. and Jaeger J.C.,"Conduction of Heat in Solids," Oxford University Press, 1959.

[11] http://www.mathworks.com/ 